\title{
A High-Accuracy High-Speed Signal Processing Circuit of Differential-Capacitance Transducers
}

\author{
Kouji Mochizuki, Member, IEEE, Kenzo Watanabe, Fellow, IEEE, and Takashi Masuda
}

\begin{abstract}
For high-accuracy signal processing of differentialcapacitance transducers, an interface circuitry is developed based on a relaxation oscillator. The interface consists of an integrator, a differentiator, and a comparator, and it uses two capacitors of the transducer-one for the integration and the other for the differentiation. This configuration allows the ratiometric operation in the amplitude domain and provides a square wave whose amplitude is proportional to the ratio of the capacitance difference between the two transducer capacitors to their sum. A circuit analysis shows that the interface can detect the capacitance change as small as $0.1 \%$ of the total capacitance in $10 \mu \mathrm{s}$. Experimental results are also given to confirm the analysis.
\end{abstract}

Index Terms-Analog circuit, capacitive transducer, intelligent transducer, pressure measurement, relaxation oscillator, signal processing.

\section{INTRODUCTION}

D IFFERENTIAL capacitance transducers consisting of ganged two capacitors are widely used for detecting pressure difference, linear displacement, acceleration, and rotational angle [1]-[3]. To extract such measurands from the complementary capacitance changes of the two capacitors, the ratiometric operation, which divides the capacitance difference between the two capacitors by their sum, is required for the interface circuit. Several techniques have so far been proposed for such a ratiometric signal processing, including switchedcapacitor (SC) analog-to-digital (A/D) [4], capacitance-tofrequency [5], capacitance-to-phase [6], and capacitance-tovoltage conversion [7], [8]. Of these, the relaxation oscillation and SC techniques are best suited for the interface because they allow a high-accuracy measurement with the simple configurations, but take a long time to perform the ratiometric operation [9], [10].

Applications of differential capacitance transducers to accelerometers require the ratiometric operation performed in a few milliseconds. Such a speed requirement can be easily met by the SC sample and hold $(\mathrm{S} / \mathrm{H})$ circuit [11], but the accuracy in ratiometric operation when the total capacitance of a transducer is a few pico Farad $(\mathrm{pF})$ is limited to $1 \%$ by clock feedthrough. Though slower in speed than the SC S/H circuit, the relaxation-oscillator based interface recently proposed also

Manuscript received May 21, 1998; revised November 9, 1998.

K. Mochizuki is with the Numazu College of Technology, Numazu, 4108501 Japan.

K. Watanabe is with the Research Institute of Electronics, Shizuoka University, Hamamatsu, 432-8011 Japan.

T. Masuda is with the Engineering Department, Yamatake-Honeywell Co. Ltd., 1- Fujisawa, 251-0015 Japan.

Publisher Item Identifier S 0018-9456(98)09812-X.

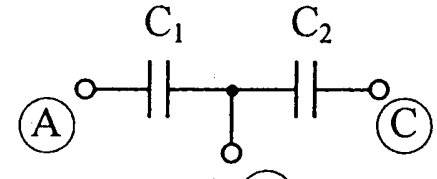

(B)

Fig. 1. An equivalent circuit of a differential capacitance transducer.

meets the speed requirement and allows much higher accuracy [12]. Using the dual-slope integration technique, the interface converts the capacitance ratio into the duty ratio. Given the capacitance ratio not in the duty ratio but in a voltage form, one can eliminate the post processing of counting and division and thereby expect much higher speed ratiometric signal processing.

Based on this idea, an interface circuitry of differential capacitance transducers is developed. In the followings, its circuit configuration, accuracy, and speed estimates, and performances of a prototype interface will be described.

\section{RATIOMETRIC SignAl Processing}

A differential capacitance transducer can be represented by two capacitors with a common electrode, as shown in Fig. 1 [8].

In a linear displacement encoder, areas of the capacitor electrodes change linearly with the displacement $x$. The capacitances $C_{1}$ and $C_{2}$ can then be expressed as follows:

$$
C_{1,2}=\frac{C_{0}}{2}(1 \pm x)
$$

where $C_{0}$ is the total capacitance of the transducer. In a differential pressure transducer, on the other hand, the spacings between the electrodes change linearly with the pressure difference $x$. Therefore, $C_{1}$ and $C_{2}$ are expressed as

$$
C_{1,2}=\frac{C_{0}}{2} \frac{1}{1 \mp x} \text {. }
$$

The capacitance change of a differential capacitance accelerometer is also given by (2).

Whether the capacitance change is linear or hyperbolic, the measurand $x$ can be detected independently of the total capacitance $C_{0}$ by the following ratiometric operation:

$$
x=\frac{C_{1}-C_{2}}{C_{1}+C_{2}} .
$$

Besides a linear detection of a measurand $x$ for easy calibration, the ratiometric operation given by (3) has another distinct 


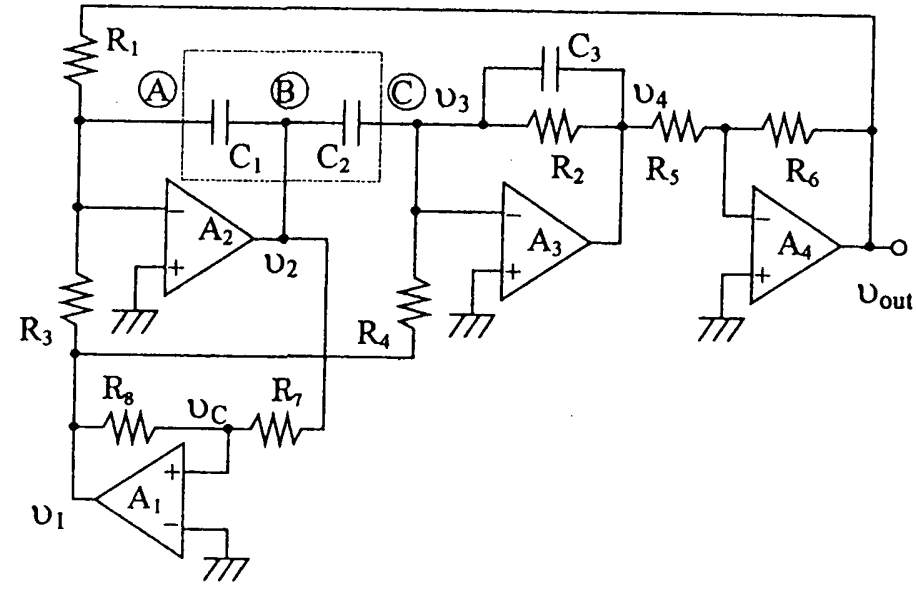

Fig. 2. The circuit diagram of the interface.

feature that the capacitance changes due to temperature, which would otherwise be a major error source are cancelled. The other error sources are stray capacitances associated with terminals (A), (B), and (C). A processing circuit should be configured such that these stray capacitances have no effect on the ratiometric operation.

\section{Circuit DescriPTION}

An interface circuit of a differential capacitance transducer is shown in Fig. 2. The core is the relaxation oscillator composed of the integrator $A_{2}$ and the comparator $A_{1}$. The differentiator $A_{3}$ and the inverting amplifier $A_{4}$ are added to the core for the ratiometric operation. Two capacitors, one $C_{1}$ for integration and the other $C_{2}$ for differentiation, represent a differential capacitance transducer. Assume that the comparator $A_{1}$ provides $+V_{\text {ref }}$ when the weighted sum of $v_{1}$ and $v_{2}$ is negative and $-V_{\text {ref }}$ when it is positive

$$
\begin{aligned}
& v_{1}(t)=V_{\text {ref }} \operatorname{sgn}\left[v_{c}(t)\right] \\
& v_{c}(t)=\frac{R_{7}}{R_{7}+R_{8}} v_{1}(t)+\frac{R_{8}}{R_{7}+R_{8}} v_{2}(t) .
\end{aligned}
$$

The output of the integrator $A_{2}$ then takes a triangular waveform and the current flowing into the differentiator takes a square waveform, as depicted in Fig. 3. The response of a differentiator to square wave excitation would be oscillatory. To avoid such an oscillatory response, $C_{3}$ is connected in parallel with $R_{2}$.

Assuming ideal op-amps and neglecting $C_{3}$, one can derive $v_{\text {out }}$ as follows:

$$
v_{c}(t)=\frac{C_{1} \frac{R_{2}}{R_{4}}-C_{2} \frac{R_{2}}{R_{3}}}{C_{1} \frac{R_{5}}{R_{6}}+C_{2} \frac{R_{2}}{R_{1}}} V_{\text {ref }} \operatorname{sgn}\left[v_{c}(t)\right] .
$$

If $R_{3}=R_{4}$ and $R_{1} R_{5}=R_{2} R_{6}$, then (6) reduces to

$$
v_{\text {out }}=V_{o} \operatorname{sgn}\left[v_{c}(t)\right]
$$

where

$$
V_{o}=\frac{R_{1}}{R_{3}} \frac{C_{1}-C_{2}}{C_{1}+C_{2}} V_{\text {ref }}=k x V_{\text {ref }}
$$

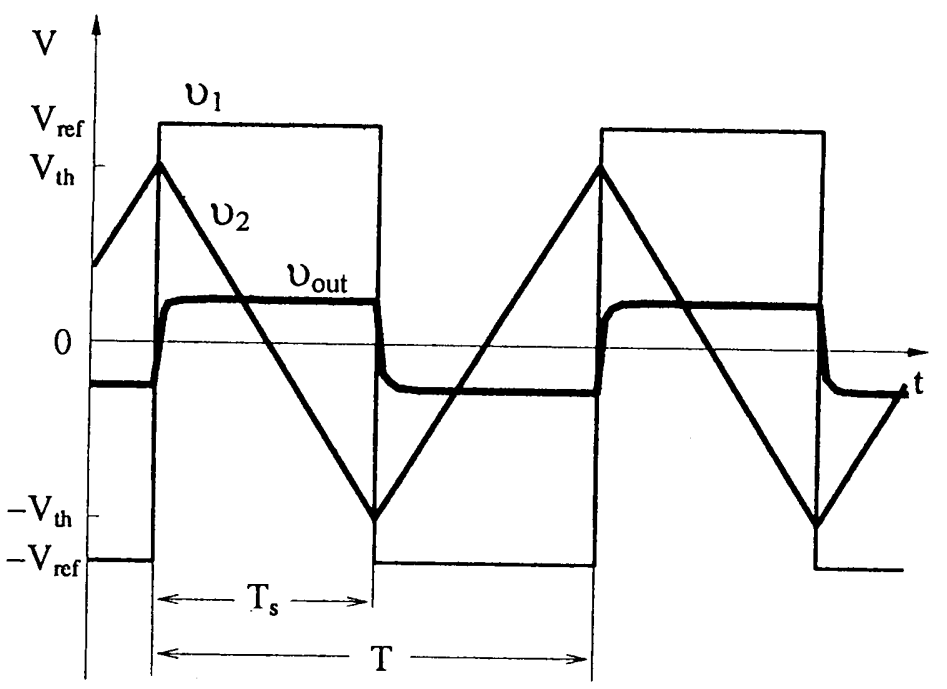

Fig. 3. Voltage waveforms in the interface.

Equation (8) indicates explicitly that the interface circuit performs the ratiometric operation in the amplitude domain. The time $T_{s}$ required for the ratiometric operation is a half period of the oscillation

$$
T_{s}=T / 2=\left(C_{1}+C_{2}\right) R_{3} .
$$

It is noted that the oscillation frequency is independent of a measurand $x$ and measures the total capacitance $C_{0}$ of a transducer.

\section{Performance Estimates}

The accuracy and speed of the ratiometric operation achievable with the interface will be estimated in this section.

The factors which affect the operational accuracy are resistance mismatches and nonideal performances of op-amps. Let $\delta_{i, j}$ be the resistance mismatch between $R_{i}$ and $R_{j}$

$$
\frac{R_{i}}{R_{j}}=1+\delta_{i, j}
$$

Substituting (10) into (6), we have to first order

$$
V_{o}=k x\left(1+\varepsilon_{R}\right) V_{\text {ref }}+\Delta V_{R}
$$

where

$$
\begin{aligned}
\varepsilon_{R} & =\frac{\delta_{3,4}+\left(\delta_{1,2}+\delta_{5,6}\right)(1+x)}{2} \\
\Delta V_{R} & =\frac{\delta_{3,4} k V_{\mathrm{ref}}}{2}
\end{aligned}
$$

are the nonlinear and offset errors due to resistance mismatches, respectively.

The finite open-loop gain $A$ and the offset voltage $V_{\text {os }}$ of an op-amp are two main error sources that affect the operational accuracy. Their effects are also described in terms of the nonlinear and offset errors as follows:

$$
V_{o}=k x\left(1+\varepsilon_{A}+\varepsilon_{V}\right) V_{\text {ref }}+\Delta V_{A}+\Delta V_{V}
$$

where $\varepsilon_{A}$ and $\Delta V_{A}$ are nonlinear and offset errors due to the finite gains and $\varepsilon_{V}$ and $\Delta V$ are those due to the offset voltages 


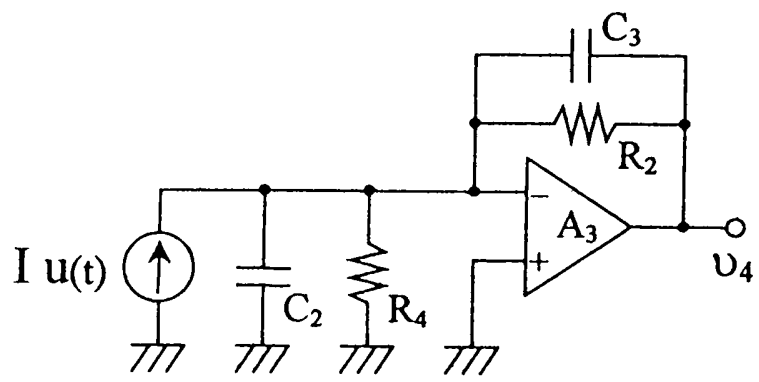

Fig. 4. The phase-compensated differentiator under step excitation.

of op-amps, respectively, given by

$$
\begin{aligned}
\varepsilon_{A} & =\frac{2}{A}(1+x) \\
\varepsilon_{V} & =\frac{R_{3}}{R_{2}} \frac{V_{\text {os }}}{V_{\text {ref }}} \\
\Delta V_{A} & =V_{\text {ref }} / A \\
\Delta V_{V} & =\left\{1+R_{1}\left(\frac{1}{R_{2}}+\frac{1}{R_{3}}\right)\right\} V_{\text {os }}
\end{aligned}
$$

In deriving the first-order expression (14), $R_{2}=R_{4}, R_{5}=$ $R_{6}, \omega C_{2} R_{2} \ll 1$, and $\omega C_{1}\left(R+1 / / R_{3}\right) \cong 1$ are assumed.

Assuming typical values $\delta=0.05 \%, A=100 \mathrm{~dB}, V_{\mathrm{os}}=$ $0.5 \mathrm{mV}, V_{\text {ref }}=10 \mathrm{~V}, R_{1}=R_{2}=R_{3}$, and $-0.5 \times 0.5$, one can evaluate each error term as follows: $\varepsilon_{R}=0.1 \%, \varepsilon_{A}=$ $3 \times 10^{-5}, \varepsilon_{V}=0.5 \times 10^{-4}, \Delta V_{R}=2.5 \mathrm{mV}, \Delta V_{A}=0.1$ $\mathrm{mV}, \Delta V_{V}=1.5 \mathrm{mV}$. The offset error can be easily nullified by an offset adjustment and thus the ultimate accuracy is determined by the nonlinear error. From the above evaluation, it is concluded that resistance matching is crucial for a high resolution.

The operational speed is limited by the differentiator because the phase compensation using $C_{3}$ is accompanied by the time delay. As described previously, a step current is applied to the inverting input terminal of op-amp $A_{3}$. The equivalent presentation of the differentiator is shown in Fig. 4 . The response $V_{4}(s)$ to the step excitation is then given by

$$
V_{4}(s)=\frac{-R_{2} I}{s} \cdot \frac{\omega_{3}}{s+\omega_{3}} \cdot \frac{\omega_{2} \omega_{u}}{s^{2}+\omega_{2} s+\omega_{2} \omega_{u}}
$$

where $\omega_{u}$ is the unity-gain bandwidth of $A_{3}$ and

$$
\begin{aligned}
& \omega_{2}=\frac{1}{C_{4} R_{4}} \\
& \omega_{3}=\frac{1}{C_{3} R_{2}} .
\end{aligned}
$$

For the phase compensation to be effective, placing $\omega_{3}$ at one tenth of $\omega_{u}$ is preferable. Let $\omega_{3}=\omega_{2} / 2=0.1 \omega_{u}$. Then, the step response $v_{4}(t)$ becomes

$$
\begin{aligned}
v_{4}(t)= & -R_{2} I\left\{1-\left(1.0-0.05 \cos 0.44 \omega_{u} t\right.\right. \\
& \left.+0.23 \sin 0.44 \omega_{u} t\right) e^{-\omega_{3} t} u(t) .
\end{aligned}
$$

The amplitudes of oscillatory terms are small owing to the phase compensation and the unit response (22) can be approximated by a simple exponential function. The $0.1 \%$ settling time $\tau_{s}$ is then given by

$$
\tau_{s}=7 \omega_{3}^{-1} .
$$

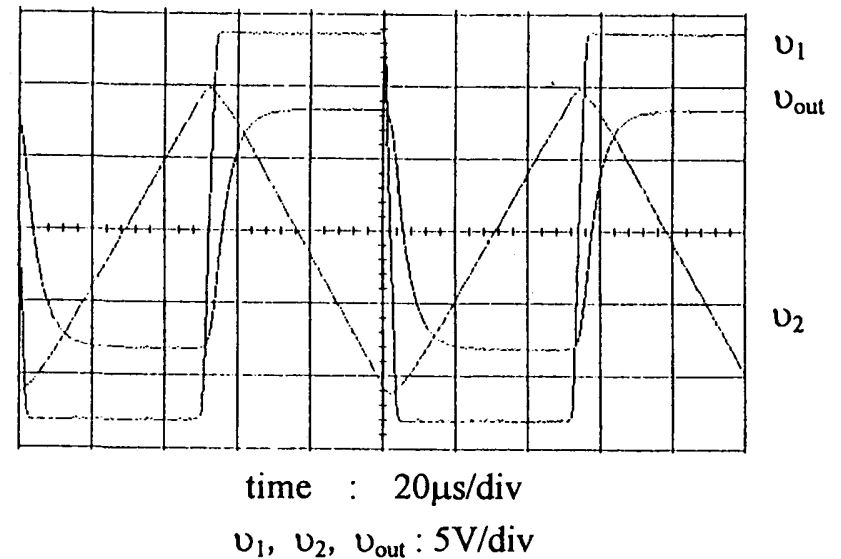

Fig. 5. Experimentally observed waveforms.

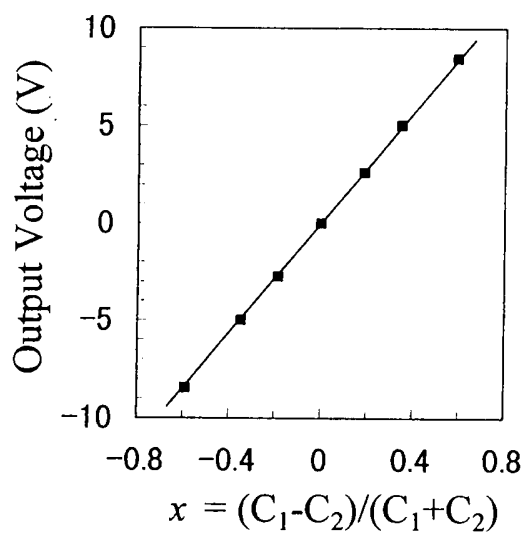

Fig. 6. The output amplitude $V_{o}$ versus $x$.

This is the minimum time required for the ratiometric operation of $0.1 \%$ accuracy if $A_{3}$ slews much faster. Specifically, if $\omega_{3}=2 \times 10^{5} \mathrm{rad} / \mathrm{s}$, then $\tau_{s}=10 \mu \mathrm{s}$.

\section{PROTOTYPE INTERFACE}

A prototype interface based on Fig. 2 was breadboarded using LF411 op-amps. The circuit parameters are $R_{1}=R_{2}=$ $R_{3}=R_{4}=1.2 \mathrm{M} \Omega, R_{5}=R_{6}=5 \mathrm{k} \Omega, C_{3}=2 \mathrm{pF}$, $V_{c c}=-V_{s s}=15 \mathrm{~V}, V_{\text {ref }}=13 \mathrm{~V}$. Mica capacitors are used for $C_{1}$ and $C_{2}$ and their values are selected such that $x$ range from -0.5 to 0.5 .

Fig. 5 shows experimentally observed waveforms when $C_{1}=20 \mathrm{pF}$ and $C_{2}=5 \mathrm{pF}(x=0.6)$. It can be seen that $v_{\text {out }}$ assumes +7.8 and $-7.8 \mathrm{~V}$ alternately and settles in $15 \mu \mathrm{s}$. These observed values agree quite well with theoretical values given by (8) and (23), respectively. The noise floor including the oscillatory amplitude is less than $2 \mathrm{mV}$. This also confirms the step response of the phase-compensated differentiator given by (22).

The amplitude $V_{o}$ of the output signal measured over the wide range of capacitance change is plotted in Fig. 6. Fig. 7 shows the output amplitude plotted for the small capacitance change. In this measurement, a ganged capacitor composed of three parallel plates whose total capacitance is $6 \mathrm{pF}$ was used in parallel with mica capacitors [7]. These preliminary results confirm the accuracy estimates in the previous section, indicating that a resolution as high as $0.1 \%$ is easily attainable with the interface. 


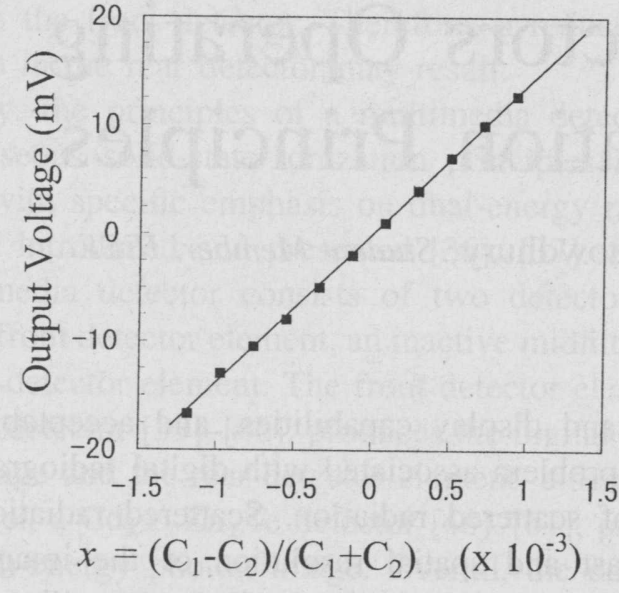

Fig. 7. The output amplitude for a small capacitance change.

\section{CONCLUSIONS}

A relaxation-oscillator-based interface circuitry has been presented, which performs the ratiometric signal processing of a differential capacitance transducer in the amplitude domain. Circuit analyses have shown that a resolution higher than $0.1 \%$ and a sampling rate as high as $100 \mathrm{ksps}$ are easily achievable with the simple configuration. A prototype interface built using off-the-shelf components has confirmed the circuit analyzes and demonstrated the validity in practical applications.

A one-chip implementation of the interface and its applications to practical transducers are future works.

\section{REFERENCES}

[1] T. Saigusa and S. Gotoh. "UNI? series electronic differential pressure transducer," Yokogawa Tech. J., vol. 22, pp. 23-29, Mar. 1978 (in Japanese)

[2] X. Li, G. C. M. Meijer, and G. W. de Jong, "An accurate smart capacitive angular-position sensor with a full circle range," in Instrum. Meas. Tech. Conf. Proc., Waltham, MA, Apr. 1995, pp. 80-82.

[3] L. K. Baxter, Capacitive Sensors Design and Applications. New York: IEEE Press, 1997.

[4] H. Matsumoto, H. Shimizu, and K. Watanabe, "A switched-capacitor change-balancing analog-to-digital converter and its application to capacitance measurement," IEEE Trans. Instrum. Meas., vol. IM-36, pp. 873-878, Dec. 1987.

[5] T. N. Toth and G. C. M. Meijer, "A low-cost smart capacitive position sensor,” IEEE Trans. Instrum. Meas., vol. 41, pp. 1041-1044, Dec. 1992.

[6] R. F. Wolffenbuttel and P. P. L. Regtien, "Capacitance-to-phase angle conversion for the detection of extremely small capacities," IEEE Trans. Instrum. Meas., vol. IM-36, pp. 868-872, Dec. 1987.

[7] K. Mochizuki, T. Masuda, and K. Watanabe, "An interface circuit for high-accuracy signal processing of differential-capacitance transducers," in Instrum. Meas. Tech. Conf. Proc., Brussels, Belgium, June 1996, pp. $1200-1203$.

[8] K. Watanabe, H. Sakai, S. Ogawa, K. Mochizuki, and T. Masuda, "High-accuracy signal processing of differential pressure transducers," 1996 IEEE Int. Workshop Emergent Technol. Instrum. Meas. (ETIM'96), Como, Italy, June 1996, pp. 111-118.
[9] T. N. Toth, G. C. M. Meijer, and H. M. M. Kerkvliet, "Ultra-linear, low-cost measurement system for multi-electrode pF-range capacitors," in Instrum. Meas. Tech. Conf. Proc., Brussels, Belgium, June 1996, pp. 512-515.

[10] Y. Cao and G. C. Temes, "High-accuracy circuit for on-chip capacitance ratio testing or sensor readout," IEEE Trans. Circuit Syst., vol. 41, pp. 637-639, Sept. 1994.

[11] H. Matsumoto and K. Watanabe, "Spike-free switched-capacitor circuits," Electron. Lett., vol. 23, pp. 428-429, Apr. 1987.

[12] K. Mochizuki, K. Watanabe, T. Masuda, and M. Katsura, "A relaxationoscillator based interface for high-accuracy ratiometric signal processing of differential-capacitance transducers," in Proc. Instrum. Meas. Tech. Conf., Ottawa. Canada, May 1997, pp. 1164-1168.

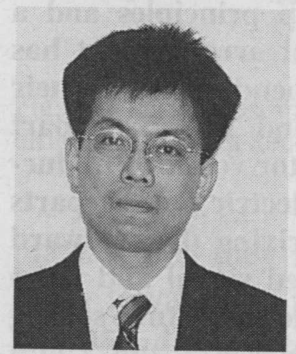

Kouji Mochizuki (M'98) was born in Shizuoka, Japan, on February 13, 1961. He received the B.E. and M.E. degrees in electronics engineering from Shizuoka University, Hamamatsu, Japan, in 1983 and 1985 , respectively.

From 1985 to 1989 , he was employed by Fujitsu Laboratories limited where he worked on epitaxial growth of III-V compound semiconductor. In July 1989 he joined Numazu College of Technology, Numazu, Japan, where he is mainly involved in resistance and capacitance measurements.

Mr. Mochizuki is a member of the Institute of Electronics, Information and Communication Engineers of Japan.

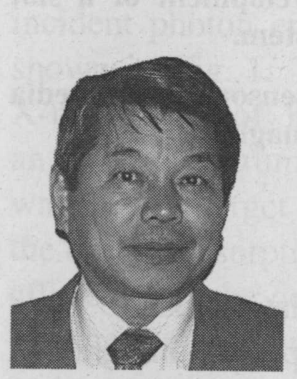

Kenzo Watanabe (M'74-SM'86-F'93) received the B.E. and M.E. degrees in engineering from Shizuoka University, Hamamatsu, Japan, in 1962 and 1966, respectively, and the Dr.Eng. degree from Kyoto University, Kyoto, Japan, in 1976.

$\mathrm{He}$ is a Professor of the Research Institute of Electronics, Shizuoka University, Hamamatsu, Japan. He has been on the faculty at Shizuoka University since 1962, serving progressively as a Research Assistant, Associate Professor, and Professor, except for a year as a Visiting Professor at University of California, los Angeles.

Dr. Watanabe serves as an AdCom member of the Instrumentation and Measurement Society is an Associate Editor of TrANSACTIONS ON INSTRUMENTATION AND MEASUREMENT and a member of the Instrumentation and Measurement Technology Conference Board of Directors.

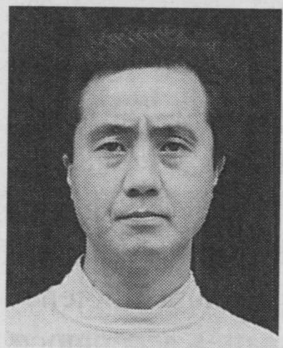

Takashi Masuda was born in Gunma, Japan, on May 5,1961 . He received the B. E. degree from the Department of Atomic Reactor Engineering, Kinki University, Osaka, Japan, in 1985. He is currently working toward the Dr.Eng. degree at the Research Institute of Electronics, Shizuoka University, Japan.

Since 1985 he has been with Yamatake Honeywell, Control Products Division, Kanagawa, Japan. Within a division of Yamatake Honeywell, he carried out several products in production sensor and sensor signal processing. 